\title{
Correlation between age and gender in Candida species infections of complete denture wearers: a retrospective analysis
}

\author{
Jolanta E Loster' \\ Aneta Wieczorek' \\ Bartłomiej W Loster ${ }^{2}$ \\ 'Department of Dental Prosthetics, \\ ${ }^{2}$ Department of Orthodontics, \\ Institute of Dentistry, Jagiellonian \\ University Medical College, \\ Kraków, Poland
}

This article was published in the following Dove Press journal:

Clinical Interventions in Aging

21 November 2016

Number of times this article has been viewed
Correspondence: Jolanta E Loster Department of Dental Prosthetics, Institute of Dentistry, Jagiellonian University Medical College, Montelupich 4, 3I-I55 Kraków, Poland

Tel +48 I2 4245555

Email jolanta.loster@uj.edu.pl
Aim: Denture-related stomatitis is a disorder that often affects denture wearers. The purpose of this study was to evaluate the intensity, genera, and frequency of yeasts in the oral cavity of complete denture wearers in terms of subject gender and age.

Materials and methods: Nine hundred twenty patients (307 males and 613 females) with complete upper dentures were selected for the study and divided into four age groups: $\leq 50$ years, $51-60,61-70$, and $>70$ years. Yeast samples were taken as a smear from the palate. The data were collected from January 15, 2007 to January 15, 2012.

Results: The distribution of the number of yeast colonies by gender was statistically significant $(P=0.02)$. Across all subjects, there was a statistically significant relationship between the intensity of yeast growth and the gender $(P=0.01)$. In every age group, the number of infection-free individuals was greater among males than females. Intermediate, intense, and abundant growth of yeast occurred most frequently in the youngest group of females.

Conclusion: The genera of Candida species and the frequency of yeast infection in denture wearers appear to be influenced by both age and gender. The complete denture wearers $\leq 50$ years of age appeared to have the greatest proclivity to oral Candida infections.

Keywords: Candida albicans, Candida species, yeasts, denture-related stomatitis

\section{Introduction}

As a result of increased access to dental care, people are retaining their natural dentition to a greater age. Nevertheless, the prevalence of edentulism remains significant, especially among the elderly. ${ }^{1}$ The population continues to age worldwide, especially in developing countries and among groups with high socioeconomic status. A World Health Organization statement from 2005 reported that $25 \%$ of Polish people aged 65-74 years were completely edentulous - a very similar situation to that in the USA, where $26 \%$ of inhabitants aged $65-69$ years were completely edentulous. ${ }^{2}$ Prosthodontic treatment with removable acrylic dentures is still frequent among older people, even in the developed world.

Denture-related stomatitis is a disorder that often affects denture wearers. ${ }^{3-5}$ A number of studies suggest that two-thirds or more of the patients who wear removable complete dentures may suffer from denture-related stomatitis. ${ }^{1,6,7}$ Despite being so common, the etiology of denture-related stomatitis is not completely understood. The current thinking is that the etiology is multifactorial but, in many cases, it includes a pathogenic response to Candida infection. ${ }^{8-10}$ Denture plaque can be a reservoir of bacteria and fungi and may be responsible for respiratory tract diseases. ${ }^{11}$ 
Our previous study showed that $58.5 \%$ of individuals wearing partial or full removable denture were affected by Candida species. ${ }^{12}$ The authors of various studies have isolated Candida species other than Candida albicans that are perhaps involved in the pathogenesis of patients with oropharyngeal candidiasis. ${ }^{13-15}$ A number of studies have compared the prevalence of denture-related stomatitis in men and women. ${ }^{1,16-18}$ Some authors have suggested that the development of denture-related stomatitis in women is higher due to hormonal changes. ${ }^{19}$

The aim of the retrospective study described here was to evaluate the intensity, genera, and frequency of yeast in the oral cavity of complete dentures wearers in terms of the gender and age of the subjects. The null hypothesis is that there are no differences in Candida species infection between male and female patients wearing complete dentures.

\section{Materials and methods}

The patient data (age, gender, general health status, length of time wearing denture, and type of denture) were collected on individual electronic medical charts using KS-Somed, an integrated software system produced by Kamsoft (Katowice, Poland). Data from January 15, 2007 to January 15, 2012 were retrospectively analyzed. The data of complete upper denture wearers who attended the Department of Prosthodontics at the University Dental Clinic in Kraków were examined with a view to their possible inclusion in the study. All of the described diagnostic and therapeutic methods were standard procedures. On the basis of our earlier study, a mycological examination was carried out on all patients with acrylic dentures in the maxilla, on the assumption that these patients were at risk of infection by Candida species. ${ }^{20}$ We excluded patients with a postoperative prosthesis in the maxilla, or who had been using the denture for $<1$ year, or who were within 1 year of oncology therapy, or who were within 3 weeks of antibiotic treatment, or who had extremely bad oral hygiene, or for whom the necessary data were lacking. The data were analyzed in terms of four age groups: $\leq 50,51-60,61-70$, and $>70$ years. Differences in data by gender were also reviewed.

The examination procedures and methods of data collection were as described in our previous reports. ${ }^{12,21}$

\section{Sample and techniques}

The research was done with the accordance of the Bioethics Committee of Jagiellonian University KBET/103/B/2004 and conformed to the criteria of The Helsinki Declaration and the International Council on Harmonisation good clinical practice guidelines. All participants signed written consent to participate in the study. The methodology of sample taking consisted of instructing the patients on what to do with their dentures in the evening and morning before attending the examination. Patients were instructed to wear the dentures overnight prior to the day of examination. Eating, drinking, smoking, and removing the denture from the mouth prior to the examination was not permitted. The examination took place between $8 \mathrm{AM}$ and $10 \mathrm{AM}$. The material was taken by smear from the palate. A sterile cotton swab was moistened with saline and rubbed on the palate between the second and third palatal folds, immediately after the removal of the upper denture. The swab was then inserted into a tube containing Steward Transport medium and sent the same day to the mycological laboratory at Kraków University Hospital.

The data from the mycological examination were examined for number of yeast colonies, their growth, and the Candida species identified. The cases were described as follows: "not infected" for 0-10 colonies, "scarce growth" for 11-20 colonies, "intermediate growth" for 21-50 colonies, "intense growth" for 51-100 colonies, and "abundant growth" for over 100 colonies. Scarce or higher growth rates resulted in a diagnosis of infection. In the analysis of the growth intensity data, intermediate and higher growth rates were considered together.

Samples showing scarce growth then were identified by colony color using CHROMagar Candida (CHROMagar, Paris, France) medium as either C. albicans or non-albicans species. In all other cases, preliminary identification of the species was made by colony color on CHROMagar Candida medium, and a commercially available validation system the Vitek 2 Compact automated identification method with YST cards (bioMerieux SA, Lyon, France) - was used from June 2009 onward to identify the non-albicans species. From January 2007 to May 2009, individual Candida species were identified using ID $32 \mathrm{C}$ testing strips, following the manufacturer's instructions (bioMerieux SA, Lyon, France). ${ }^{12}$

The number of Candida species colonies for each patient was evaluated. The distribution of the genders by age groups, the intensity of yeast growth, the distribution of the number of yeast colonies, and the distribution of Candida species morphotypes were then determined.

\section{Statistical analysis}

The data were analyzed using a statistical program (SPSS Statistics 17.0 for Windows 2008). The normality of the distribution was evaluated using the Kolmogorov-Smirnov 
and Shapiro-Wilk tests. The Pearson chi-square test, the Mann-Whitney $U$-test, and the Kruskal-Wallis test were used to examine the differences between the groups; $P$-values $<0.05$ were considered statistically significant.

\section{Results}

\section{Distribution of genders by age groups}

Over the 5-year period, 920 complete denture wearers (307 males and 613 females) who did not match the exclusion criteria were incorporated in the analysis.

The results of the Kolmogorov-Smirnov and ShapiroWilk tests for the normality of the age distribution were statistically significant $(P<0.001)$, meaning that the data were not from a normally distributed population and that nonparametric tests should be used.

Patient ages ranged from 25 to 88 years (males from 44 to 88 and females from 25 to 88 , respectively), with a mean age of 65.7 years (males 67.7 and females 64.7). The difference in ages between sexes was statistically significant $(P<0.001)$ according to the Mann-Whitney $U$-test.

Table 1 presents the distribution of gender by age groups. The difference is statistically significant $(P<0.001)$. In each age group, the number of females was greater by a factor of three in the two youngest groups.

\section{Intensity of yeast growth}

Diagnoses of yeast infection were made for 542 of 920 patients (58.8\%), of which 161 were males $(52.4 \%)$ and 381 were females $(62.2 \%)$, respectively. The intensity of yeast growth by gender is shown in Figure 1. The difference between males and females was statistically significant $(P=0.006)$.

Table I The distribution of gender in age groups

\begin{tabular}{llll}
\hline Age groups (years) & Male & Female & Total \\
\hline$\leq 50$ & & & \\
$\quad \begin{array}{l}\text { Number } \\
\% \text { of gender }\end{array}$ & 15 & 50 & 65 \\
$>50 / \leq 60$ & 4.9 & 8.2 & 7.1 \\
$\quad \begin{array}{l}\text { Number } \\
\% \text { of gender }\end{array}$ & 51 & 165 & \\
$>60 / \leq 70$ & 16.6 & 26.9 & 216 \\
$\quad \begin{array}{l}\text { Number } \\
\% \text { of gender }\end{array}$ & 113 & 208 & 23.5 \\
$>70$ & 36.8 & 33.9 & 321 \\
$\quad \begin{array}{l}\text { Number } \\
\% \text { of gender }\end{array}$ & 128 & 190 & 34.9 \\
Total & 41.7 & 31.0 & 318 \\
$\quad \begin{array}{l}\text { Number } \\
\% \text { of gender }\end{array}$ & 307 & 613 & 34.6 \\
\hline
\end{tabular}

Note: Pearson's chi-square, $P<0.001$.

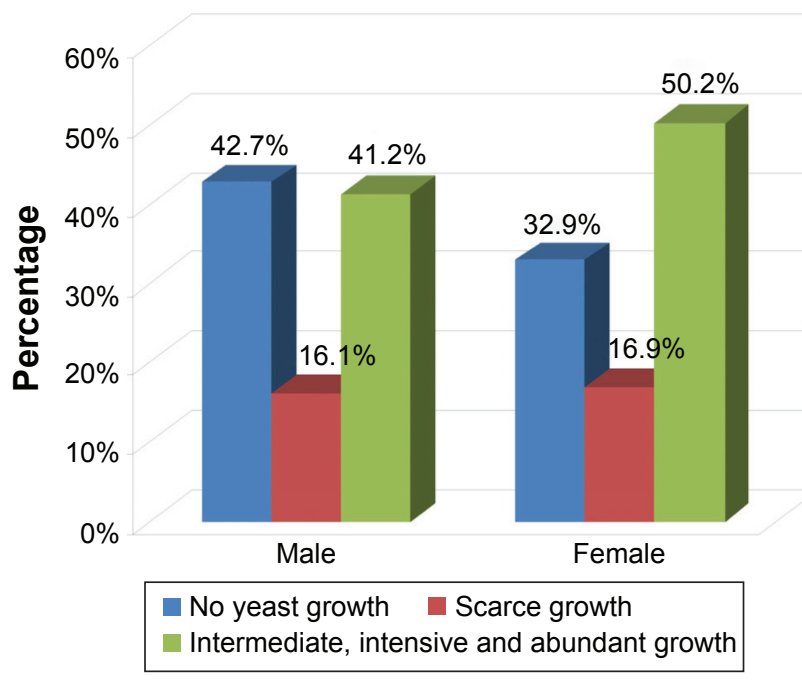

Figure I Yeast growth by gender (Pearson's chi-square, $P=0.006$ ).

The intensity of yeast growth was assessed by patient age, with statistical analysis showing significant differences $(P=0.002)$. In the youngest group of patients (those $\leq 50$ years of age), the number of patients without a yeast infection was much smaller than in the other groups. The greatest percentage of intermediate, intense, and abundant growths of yeast are seen in this age group. The results are shown in Figure 2.

Data on the intensity of yeast growth with respect to age groups in females and males were analyzed. Males did not differ significantly in terms of intensity of yeast growth across the age groups $(P=0.178)$. In the female group,

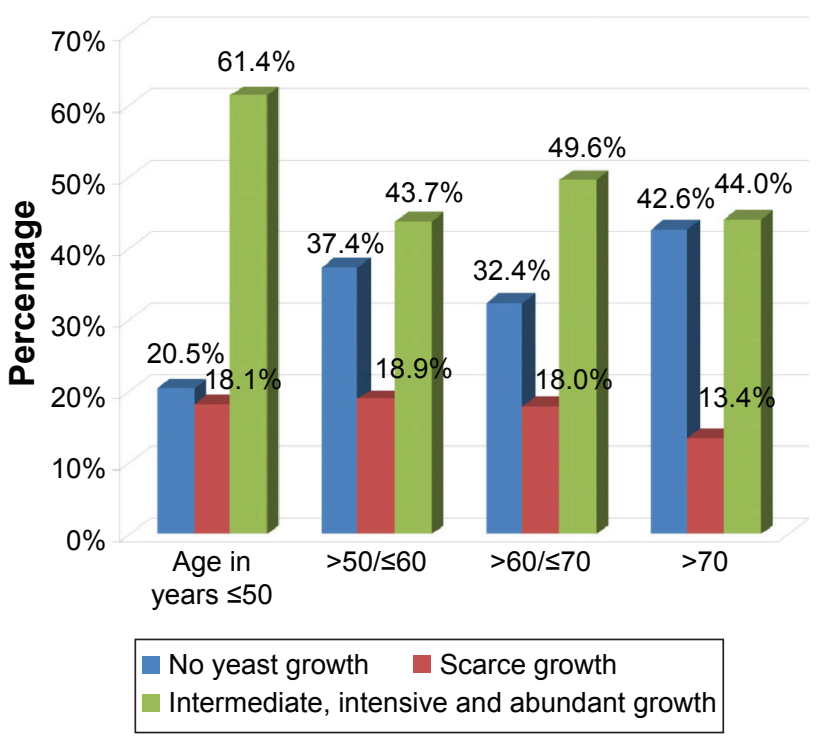

Figure 2 Yeast growth in each age group (Pearson's chi-square, $P=0.002$ ). 
the difference was statistically significant $(P=0.005)$. The smallest percentage of uninfected females was found in the youngest group and the greatest percentage was seen in the oldest group. Scarce growth of yeast was most frequent in females aged 50-60 years. Intermediate, intense, and abundant growth of yeast occurred most frequently in the youngest group of females. These results are presented in Figure 3.

A correlation of gender with each age group has been made. The results are shown in Figure 4. A statistically significant difference between males and females in the intensity of yeast growth was found in the 61- to 70-yearold group $(P=0.029)$. In this group, the percentage of males with scarce yeast growth was greater than that of females, and the percentage of females with intermediate, intense, or abundant growth of yeast was greater than in males. There were no statistically significant differences between the sexes in the other age groups.

\section{Distribution of yeast colonies counts}

One Candida species colony was found in 421 patients (45.8\%), two colonies in 116 patients $(12.6 \%)$, and three colonies in five patients $(0.5 \%)$. For further analysis, we combined the patients with two or three Candida colonies into one group. The distribution of the number of yeast colonies by gender is presented in Figure 5. The difference was statistically significant $(P=0.017)$. In both categories of infection (one colony and two or more colonies), the percentage of females was higher than the percentage of males.
The next evaluation concerned the distribution of the number of Candida species colonies based on age groups. The results are presented in Figure 6. Statistical analysis showed a significant difference $(P<0.001)$.

An assessment was then made of the relative numbers of yeast colonies in males and females with respect to age. Males did not differ significantly in terms of the number of Candida colonies in either age group $(P=0.313)$. However, in the female group, the differences were statistically significant $(P=0.021)$. Among those $\leq 50$ years of age, $28 \%$ of females had two or more yeast colonies (the highest number). In the next two age groups examined, one colony was detected in $>50 \%$ of females. In the oldest age group, the highest number of females did not have any yeast infection. The results are shown in Figure 7.

Finally, correlations between the number of colonies, gender, and age were calculated. In all age groups, the males and females did not differ significantly in the distribution of the number of Candida colonies. The significance values were $P=0.759$ in the youngest group, $P=0.263$ in the next age range, $P=0.149$ in the second oldest group, and $P=0.39$ in the oldest group.

\section{The distribution of Candida species morphotypes}

In the course of this survey, 22 morphotypes of Candida species were collected from the participants' palates. The difference in the distribution of Candida species across patient sex and age was not statistically significant (Pearson's chi-square

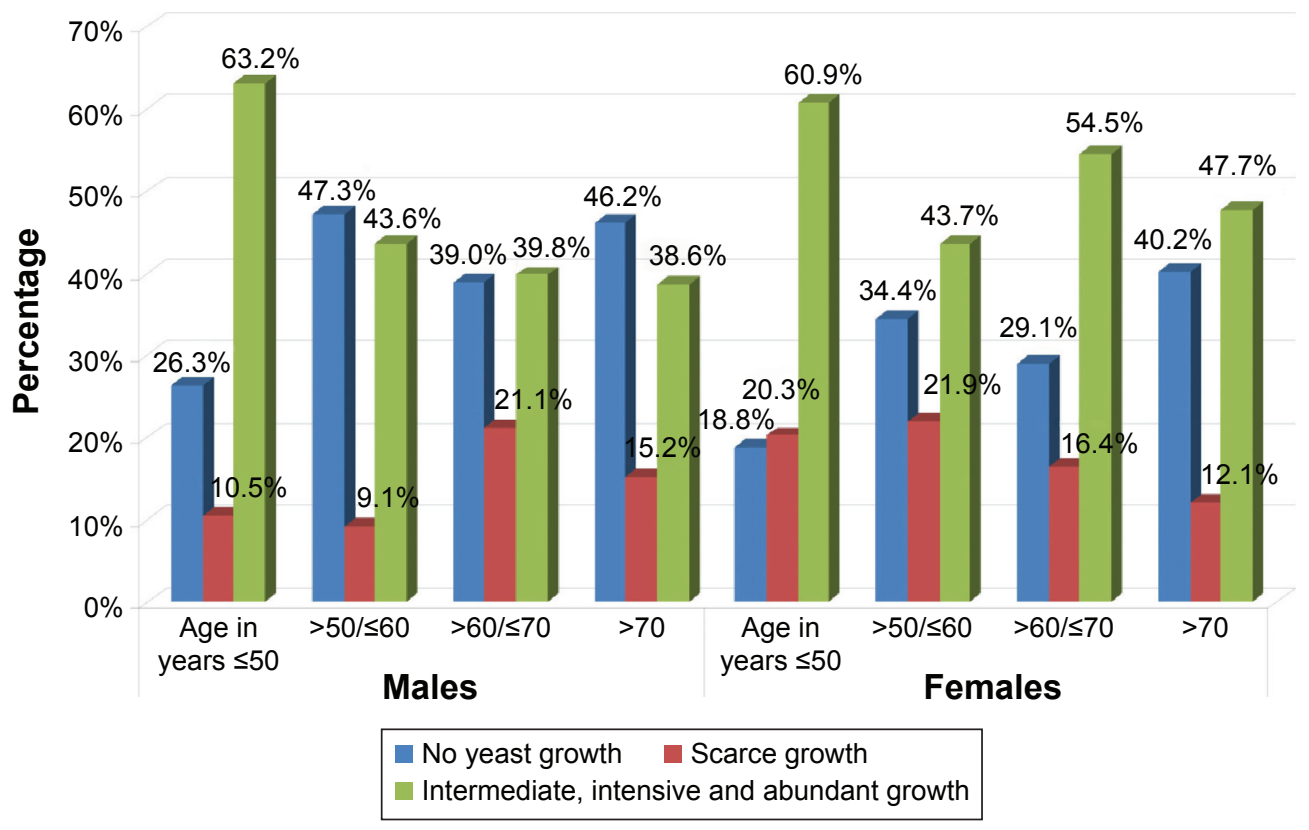

Figure 3 Yeast growth in each age group for males $(P=0.178)$ and females $(P=0.005)$ separately (Pearson's chi-square test). 


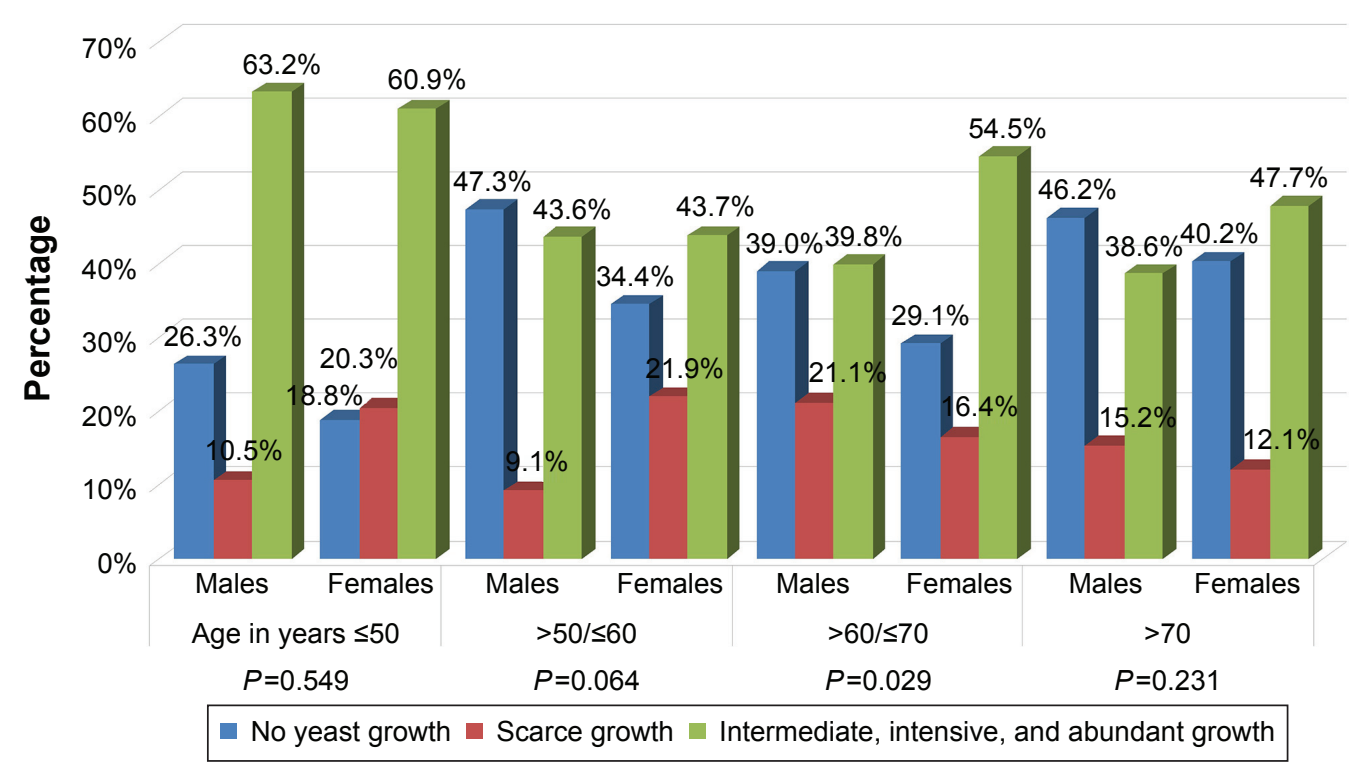

Figure 4 Yeast growth in each age group between genders (Pearson's chi-square test).

test, $P=0.590$ and $P=0.109$, respectively). $C$. albicans was the most common species found in both sexes. The distribution of the types of yeasts most frequently detected in males and females is shown in Figure 8.

The most frequently detected yeasts were C. albicans, Candida glabrata, and Candida tropicalis. These species occurred in respectively, $60.8 \%, 20.5 \%$, and $4.8 \%$ of all affected patients. Other yeast species were present in frequencies of $<1.3 \%$.

The statistical analysis did not disclose significant differences in relation to gender in the distribution of these three most frequently occurring yeasts in the different age groups ( $P=0.912$ for males and $P=0.223$ for females).

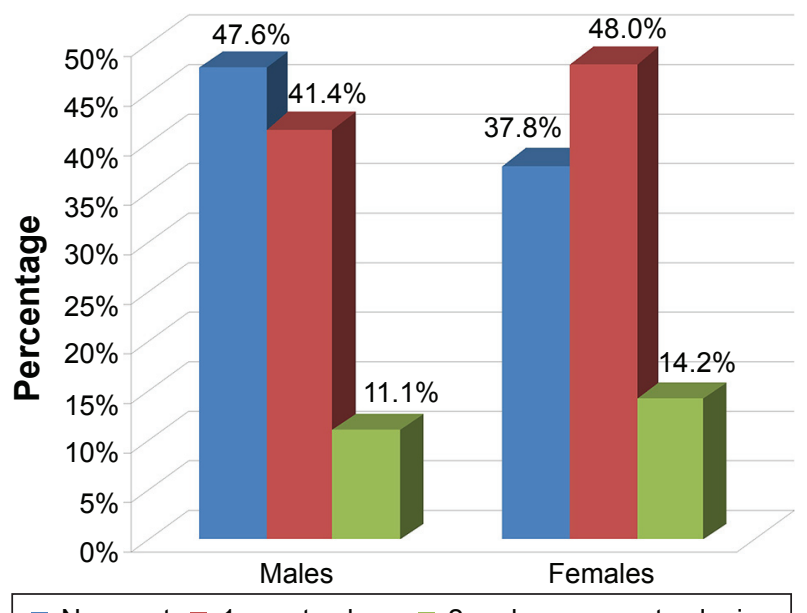

No yeast $\square 1$ yeast colony $\square 2$ and more yeast colonies

Figure 5 Number of Candida species colonies, by gender (Pearson's chi-square, $P=0.017$ ).
In each age group, the distribution of Candida species with respect to gender was examined, but was not statistically significant for the age groups. The only interesting finding was that females below and equal to 50 showed the greatest frequency of Candida species other than C. albicans, C. glabrata, and $C$. tropicalis (23.1\% versus $7.1 \%$ in males).

\section{Discussion}

The use of removable prosthetic appliances is commonly associated with denture-related stomatitis, particularly in the upper arch. ${ }^{9,22}$ There are many factors that influence the severity of this disease, including continuous denture wearing, denture plaque, bad fitting and occlusal instability, general health problems like diabetes or hypertension, and the age of the denture. Regardless of these problems, Candida species are the most frequently diagnosed. ${ }^{1,10,16,17,19}$ In our examination, a smaller percentage of the females were not infected by yeast, and the intensity of growth was greater in the female group than among the males, presenting microbiologically as intermediate, intense, and abundant growths of yeast. We also noticed that, in the youngest group of patients (those $\leq 50$ years of age), the number of uninfected patients was smallest, and was even smaller than the number of subjects with two or more yeast colonies in the oral cavity.

There are some studies that report a greater prevalence of denture-related stomatitis in men than in women. The literature review by Gendreau and Loewy shows that, in a study conducted in Slovenia, Finland, and Chile, the number of female denture users was greater than the number of males. ${ }^{1}$ In our report, the number of women wearing an upper full denture was 


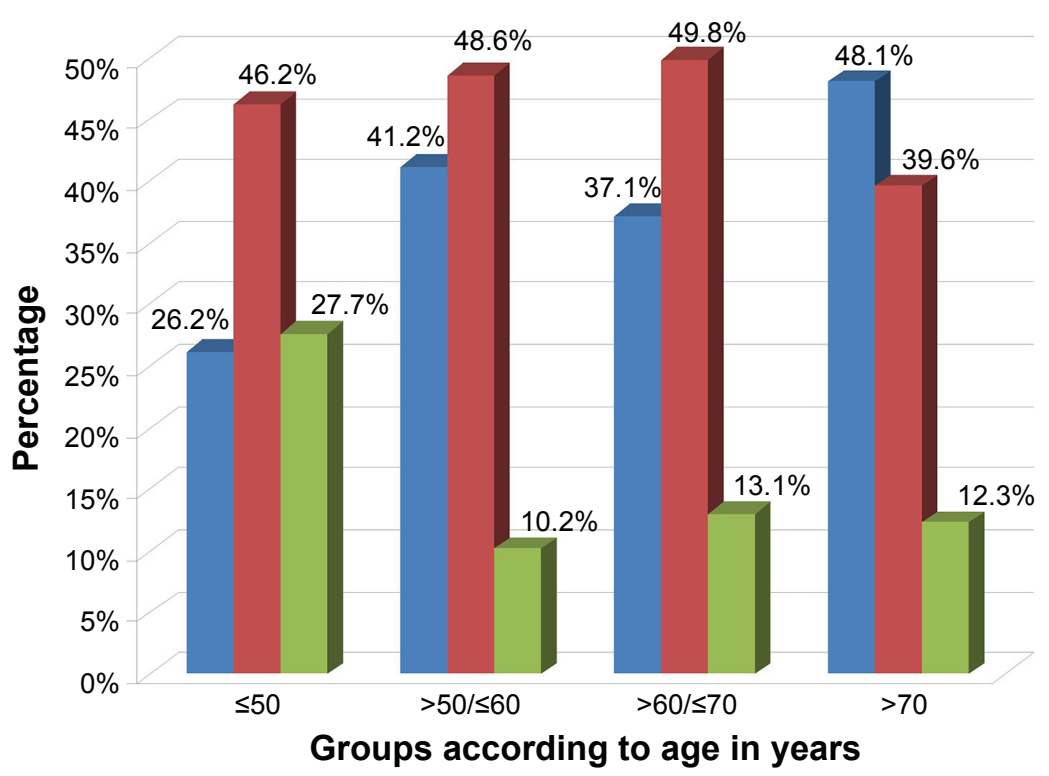

No yeast $\square 1$ yeast colony $\square 2$ and more yeast colonies

Figure 6 Distribution of Candida species colony numbers in each age group (Pearson's chi-square, $P<0.00 \mathrm{I}$ ).

also greater than the number of men in each of the age groups analyzed. A similar relation was reported by Jainkittivong et $\mathrm{al},{ }^{5}$ Abaci et al, ${ }^{16}$ and Figueiral et al, ${ }^{17}$ but in other studies, the results were the opposite. ${ }^{3}$ More detailed studies should be carried out to determine the reason for this difference in gender prevalence, possibly in psychosocial and dental terms.

In our study, when the number of yeast colonies in the whole group was examined, significant gender differences were seen. When the examined group was divided by age, significant differences were also found. This means that a difference between males and females does occur, but that various levels of yeast infection also occurs at different ages. It is especially noticeable that, in the female group, the number of yeast colonies changes with age group. No statistical differences were found between males and females when the number of yeast colonies present in the different age groups was considered. Similar results were obtained for calculating the intensity of the growth of yeast. The only gender difference between age

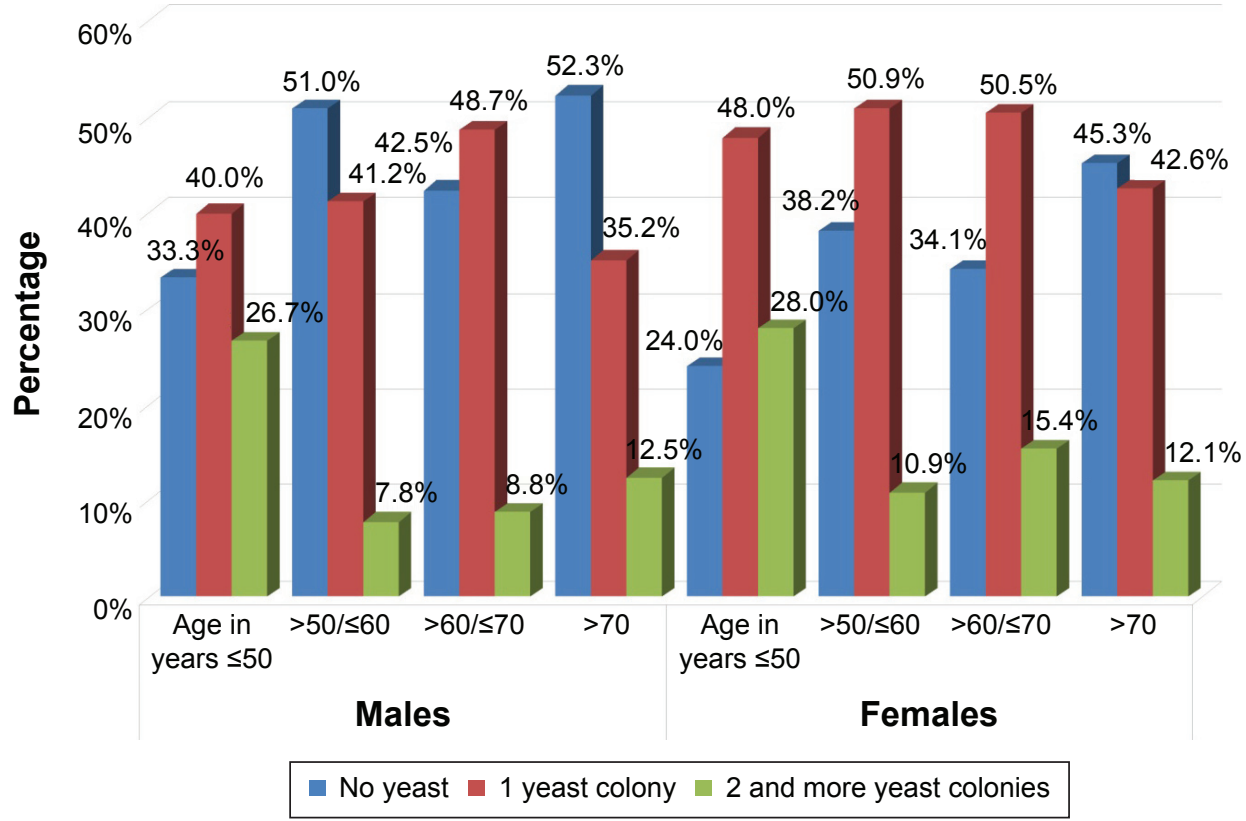

Figure 7 Number of Candida species colonies in each age group separately for males $(P=0.3 \mid 3)$ and females (Pearson's chi-square test, $P=0.02 I)$. 

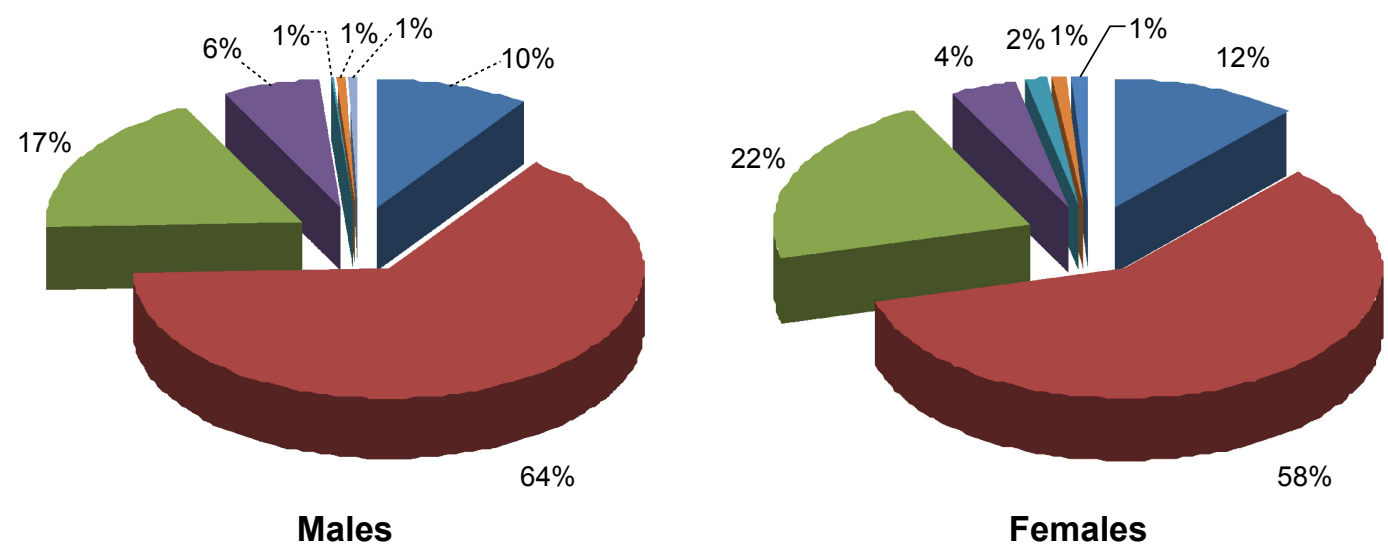

Other yeasts $\square$ Candida albicans $\square$ Candida glabrata $\square$ Candida tropicalis
Candida parapsilosis $\quad$ Candida inconspicua $\quad$ Candida krusei

Figure 8 Most frequently detected Candida species in males and females.

groups was related to the $61-70$ years of age group. There were significantly more females with intermediate, intense, and abundant growth of yeast than males. This shows that drawing conclusions from groups of patients with a broad spread of ages can lead to misinterpretation of results. This could apply, for example, to the work of Figueiral et al where the groups of individuals involved were $<35$ years of age, between 35 and 75 years, and $>75$ years. ${ }^{17}$ Our research has concluded that, in each age group, the number of uninfected females was smaller than the number of males. Patients wearing complete dentures under and equal to the age of 50 suffered from more infection and had a greater diversity of infecting species. Dentists should therefore pay special attention to the condition of the palatal mucosa of all complete denture wearers, especially if the denture has been used for many years.

Our investigation of Candida species from the oral cavity of full denture wearers showed that C. albicans, C. glabrata, and C. tropicalis were most common. C. albicans was found in $60.8 \%$ of our complete denture users, and there were no statistical differences between genders; these results are similar to those of other researchers, ${ }^{4,15}$ though some reports have described a greater frequency of C. albicans., ${ }^{3,16,17}$ In our earlier article, which involved patients with complete and partial acrylic dentures in the upper jaw, the percentage with C. albicans was $64.9 \%{ }^{12}$ When we examined separate age groups in the current study, we found that the distribution between gender and age of the percentage of $C$. albicans in complete denture wearers varied from $42.3 \%$ in the youngest group of females to $72.4 \%$ in males aged $51-60$ years. We noticed a statistically insignificant, but nevertheless important finding, in the youngest group of females in relation to the yeast species present: this group had the greatest number of Candida species, other than C. albicans, C. glabrata, and C. tropicalis. However, it should be noted that the methods used to examine the yeast varied in different studies. Some results come from saliva specimens, while others are from swabbing the dorsum of the tongue, the denture, or the palate. We conducted swab examinations of the mucosa of palate, this being the location of the infection connected with dentures. Also, different amounts of yeast have been obtained from different locations in the same patient. ${ }^{16}$ The comparison of results may therefore be unreliable.

On the basis of our research, it has been possible to reject the null hypothesis that there were no differences in Candida species infection between the sexes in patients wearing complete dentures. However, if the group of patients examined is congruous and includes those of similar ages (within a 10-year span), it is not possible to reject the hypothesis.

\section{Conclusion}

Yeast infections in the oral cavity of full denture wearers are common, but the genera of yeasts and their frequencies appear to be influenced by both age and gender. With regard to the patients in our study population, complete denture wearers of $\leq 50$ years of age appeared to have the greatest proclivity for oral candidiasis infections. Researchers should pay special attention to age when selecting patients with complete dentures for investigation.

\section{Acknowledgments}

The authors thank Jolanta Kędzierska, PhD, MS in biology, and Anna Kujawska, MS, for mycological procedure support. 
Publication was supported by the Faculty of Medicine of Jagiellonian University Medical College (Leading National Research Centre 2012-2017).

\section{Disclosure}

The authors report no conflicts of interest in this work.

\section{References}

1. Gendreau L, Loewy ZG. Epidemiology and etiology of denture stomatitis. J Prosthodont. 2011;20(4):251-260.

2. Petersen PE, Yamamoto T. Improving the oral health of older people: the approach of the WHO Global Oral Health Programme. Community Dent Oral Epidemiol. 2005;33(2):81-92.

3. Dar-Odeh NS, Shehabi AA. Oral candidosis in patients with removable dentures. Mycoses. 2003;46(5-6):187-191.

4. Zaremba ML, Daniluk T, Rozkiewicz D, et al. Incidence rate of Candida species in the oral cavity of middle-aged and elderly subjects. Adv Med Sci. 2006;51(Suppl 1):233-236.

5. Jainkittivong A, Aneksuk V, Langlais RP. Oral mucosal lesions in denture wearers. Gerodontology. 2010;27(1):26-32.

6. Webb BC, Thomas CJ, Willcox MD, Harty DW, Knox KW. Candidaassociated denture stomatitis. Aetiology and management: a review. Part 1. Factors influencing distribution of Candida species in the oral cavity. Aust Dent J. 1998;43(1):45-50.

7. Ercalik-Yalcinkaya S, Ozcan M. Association between oral mucosal lesions and hygiene habits in a population of removable prosthesis wearers. J Prosthodont. 2015;24(4):271-278.

8. Budtz-Jorgensen E. The significance of Candida albicans in denture stomatitis. Scand J Dent Res. 1974;82(2):151-190.

9. Wilson J. The aetiology, diagnosis and management of denture stomatitis. Br Dent J. 1998;185(8):380-384.

10. Zomorodian K, Haghighi NN, Rajaee N, et al. Assessment of Candida species colonization and denture-related stomatitis in complete denture wearers. Med Mycol. 2011;49(2):208-211.
11. Przybylowska D, Mierzwinska-Nastalska E, Swoboda-Kopec E, Rubinsztajn R, Chazan R. Potential respiratory pathogens colonisation of the denture plaque of patients with chronic obstructive pulmonary disease. Gerodontology. 2016;33(3):322-327.

12. Loster BW, Loster J, Wieczorek A, Ryniewicz W. Mycological analysis of the oral cavity of patients using acrylic removable dentures. Gastroenterol Res Pract. 2012;2012:951572.

13. Li L, Redding S, Dongari-Bagtzoglou A. Candida glabrata: an emerging oral opportunistic pathogen. J Dent Res. 2007;86(3):204-215.

14. Lyon JP, da Costa SC, Totti VM, Munhoz MF, de Resende MA. Predisposing conditions for Candida spp. carriage in the oral cavity of denture wearers and individuals with natural teeth. Can J Microbiol. 2006; 52(5):462-467.

15. Daniluk T, Tokajuk G, Stokowska W, et al. Occurrence rate of oral Candida albicans in denture wearer patients. Adv Med Sci. 2006; 51(Suppl 1):77-80.

16. Abaci O, Haliki-Uztan A, Ozturk B, Toksavul S, Ulusoy M, Boyacioglu H. Determining Candida spp. incidence in denture wearers. Mycopathologia. 2010;169(5):365-372.

17. Figueiral MH, Azul A, Pinto E, Fonseca PA, Branco FM, Scully C. Denture-related stomatitis: identification of aetiological and predisposing factors - a large cohort. J Oral Rehabil. 2007;34(6):448-455.

18. Freitas JB, Gomez RS, De Abreu MH, Ferreira EFE. Relationship between the use of full dentures and mucosal alterations among elderly Brazilians. J Oral Rehabil. 2008;35(5):370-374.

19. Webb BC, Thomas CJ, Whittle T. A 2-year study of Candida-associated denture stomatitis treatment in aged care subjects. Gerodontology. 2005; 22(3):168-176.

20. Loster BW, Majewski S, Macura AB, Ziemniak W. Infection of the oral cavity by a fungus of the genus Candida in complete denture users vs oesophageal candidiasis. Mikologia Lekarska. 2007;14(3):157-161.

21. Majewski S, Loster BW, Wiśniewska G. Diagnostic and therapeutic procedure in cases of prosthetic stomatitis - based on their own research and long-time clinical observations. Implantoprotetyka. 2003;427-434.

22. Coco BJ, Bagg J, Cross LJ, Jose A, Cross J, Ramage G. Mixed Candida albicans and Candida glabrata populations associated with the pathogenesis of denture stomatitis. Oral Microbiol Immunol. 2008; 23(5):377-383.
Clinical Interventions in Aging

\section{Publish your work in this journal}

Clinical Interventions in Aging is an international, peer-reviewed journal focusing on evidence-based reports on the value or lack thereof of treatments intended to prevent or delay the onset of maladaptive correlates of aging in human beings. This journal is indexed on PubMed Central, MedLine,

\section{Dovepress}

CAS, Scopus and the Elsevier Bibliographic databases. The manuscript management system is completely online and includes a very quick and fair peer-review system, which is all easy to use. Visit http://www.dovepress. com/testimonials.php to read real quotes from published authors. 